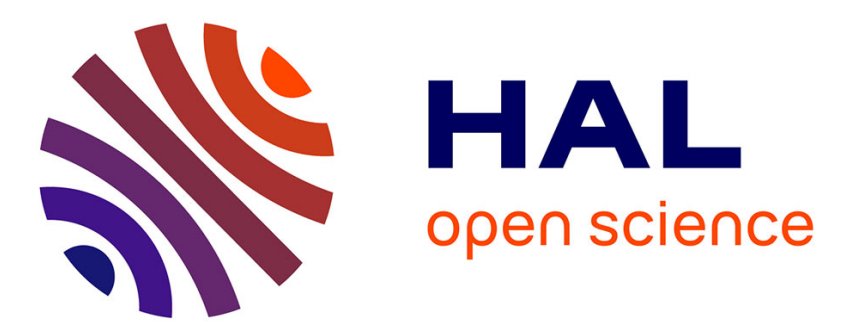

\title{
A Microfabricated Planar Digital Microrobot for Precise Positioning Based on Bistable Modules
}

\author{
Vincent Chalvet, Yassine Haddab, Philippe Lutz
}

\section{To cite this version:}

Vincent Chalvet, Yassine Haddab, Philippe Lutz. A Microfabricated Planar Digital Microrobot for Precise Positioning Based on Bistable Modules. IEEE Transactions on Robotics, 2013, 3, pp.641-649. hal-00840149

\section{HAL Id: hal-00840149 \\ https://hal.science/hal-00840149}

Submitted on 1 Jul 2013

HAL is a multi-disciplinary open access archive for the deposit and dissemination of scientific research documents, whether they are published or not. The documents may come from teaching and research institutions in France or abroad, or from public or private research centers.
L'archive ouverte pluridisciplinaire HAL, est destinée au dépôt et à la diffusion de documents scientifiques de niveau recherche, publiés ou non, émanant des établissements d'enseignement et de recherche français ou étrangers, des laboratoires publics ou privés. 


\title{
A Microfabricated Planar Digital Microrobot for Precise Positioning Based on Bistable Modules
}

\author{
Vincent Chalvet, Yassine Haddab, Member, IEEE and Philippe Lutz, Member, IEEE
}

\begin{abstract}
Size reduction is a constant objective in new technologies, for which very accurate devices are needed when manipulating sub-millimetric objects. A new kind of microfabricated microrobot based on the use of bistable modules is designed to perform open-loop controlled micropositioning tasks. The DiMiBot (Digital MicroroBot) opens a new paradigm in the design of microrobots by using mechanical stability instead of complex control strategies. We propose a new architecture of digital microrobot for which forward and inverse kinematics models are easy to use. These kinematic models are validated with FEA simulations before the fabrication of a real DiMiBot prototype. Tests and characterization of the prototype are made and compared to the desired behavior. Thanks to its submicrometric resolution and to its small dimensions $(\sim 400 \mu \mathrm{m}$ thickness), it is able to manipulate micro-objects in confined environments, where no other robot can be used.
\end{abstract}

Index Terms-Micro/Nano Robots, Mechanism Design, Flexible Arms, Bistable Module, Discrete Workspace

\section{INTRODUCTION}

In order to meet the needs of microcomponents manipulation and assembly, significant research activities have been performed in the field of microrobotics which deals with the design, fabrication and control of microrobots. These microrobots are intended to perform various tasks in the Microworld ( i.e. the world of submillimetric objects), in particular micromanipulation tasks of single objects (mechanical or biological) as positioning, characterizing or sorting as well as industrial micro-assembly. Achieving efficient robotic tasks at this scale remains a great challenge and requires submicrometric resolution and accuracy in order to interact with micrometric objects. Methods and strategies used to build conventional robots are often not applicable in the microworld. New mechatronic approaches, new actuators and robot kinematics are needed. Researches done in the world have shown that the use of active materials to actuate microrobots gives better performances than the use of more traditional actuators. Piezoelectric materials, shape memory alloys (SMA) and active polymers have been successfully used to actuate various types of microrobots. However, despite their intrinsic high resolution, these active materials present some disadvantages, making the design of efficient controllers a hard task [1]. Their behavior is often complex, nonlinear and sometimes non stationary. Closed-loop control of the microrobots requires the design and the integration of very small sensors and the use of bulky and expensive instruments for signal processing and

V. Chalvet, Y. Haddab and P. Lutz are with the Automatic Control and Micro-Mechatronic Systems Department, FEMTO-ST Institute, Besançon, 25000 France email: \{vincent.chalvet;yassine.haddab;philippe.lutz\}@femtost.fr .

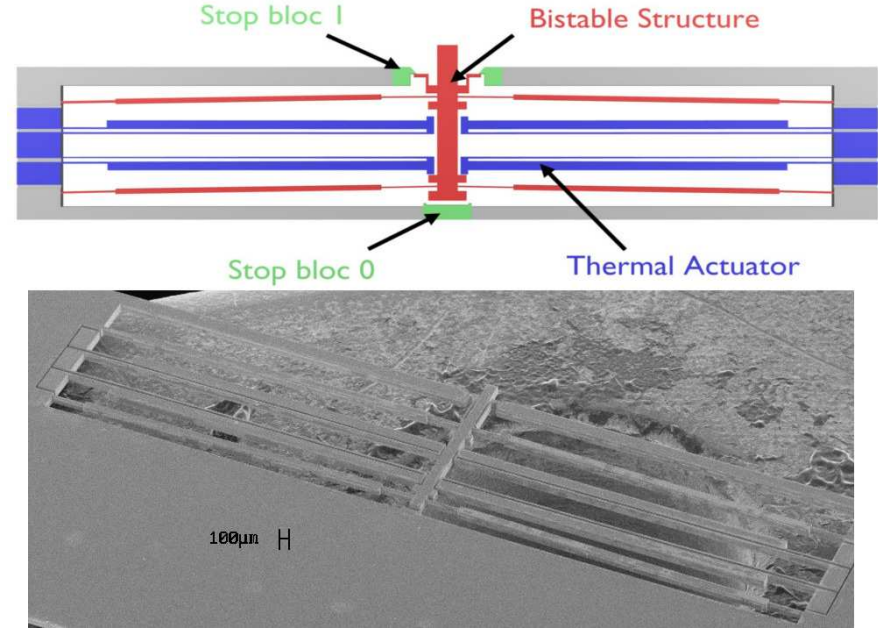

Fig. 1. One Bistable Module: CAD model (top) and Scanning Electron Microscope (SEM) image (bottom)

real-time operating. Packaging and integration of the sensors and actuators is also a hard problem. This is why building multi-degrees of freedom microrobots able to perform complex tasks is difficult. Moreover, in many cases, the size of the robot itself should be very small in order to manipulate microobjects in confined environments. The design of a microrobot contains two parts: the design of end-effector to interact with the manipulated micro-objects and the design of microrobot arm used to move the end-effector in the working area. While many research activities have been performed to develop endeffectors compatible with the microworld [2], [3], few works concerned the development of microrobot arms adapted to the microworld. Most of current robots are bulky and based on the miniaturization of traditional kinematics, such as anthropomorphic robots (Kleindiek's MM3A [4]) or delta robots (Asyril's Pocket Delta [5]). Their size is not really compatible with the microworld and limits considerably the use of microrobots to execute complex tasks in confined environments. Other planar stages better suited for micropositioning tasks use active materials as actuators ([6], [7]) but are also hardly used in confined environments because of the need of sensors.

In [8], [9] we have proposed a new paradigm in building microrobots, named digital microrobots. These studies were dedicated to the design of the bistable modules. On this paper we propose an original design of digital microrobot based on the use of the bistable modules designed in [8], [9], combined with a flexible structure, able to generate a two dimensional workspace. The bistable module is composed 


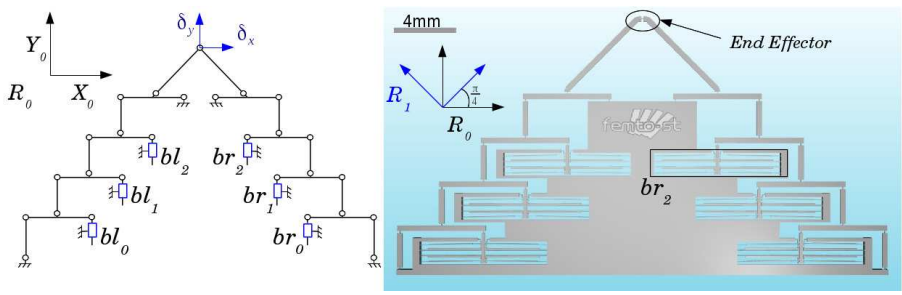

Fig. 2. Robot Design: Kinematic scheme (left) and CAD model (right)

of three elements: a mechanical bistable structure, two pairs of electro-thermal actuators and two stop-blocks. The electrothermal actuators push forth and back the bistable structure, one pair of actuators pushing in one direction, to reach each stable position. The stop-blocks limit the displacement of the bistable structure between the two positions, and also give the robustness of each position by adding a blocking force. In this paper, the two robust positions are separated by $30 \mu \mathrm{m}$ and the blocking force is $1.54 \mathrm{mN}$. As it is mechanically stable, the module does not need any power supply to stay in a given position. Power is only needed when switching between states.

In this paper, we propose an original digital microrobot design. We first introduce the specifications used for this microrobot before presenting the chosen design and the calculation of its kinematics models. Section $I V$ presents the dimensioning study of this robot before dealing with microfabrication process and characterization of a digital microrobot.

\section{The DiMiBot}

\section{A. Specifications for a Digital Microrobot}

Open-loop controllable manipulators were already studied in the macroscale robotics domain with the VGT (Variable Geometry Truss) manipulator in [10] which is a light-weight hyper-redundant two dimensional manipulator based on the use of binary actuators. Because of the high redundancy of this robot, complex forward and inverse kinematics models were developed in [11], [12]. Another three dimensional digital manipulator was also developed in [13], the BRAID (Binary Robotic Articulated Intelligent Device) for space usage purpose. The kinematic architectures used for these robots are not applicable to the design of robots operating at microscale. Assembled architectures are indeed not well adapted to microrobotics because of many drawbacks really troublesome for precise micromanipulation tasks (friction, backlash, ....).

Specifications for the design of the microrobot are mainly dictated by common microworld requirements. However, these specifications must be consistent with microfabrication technology limitations. In order to work in confined environments, the microrobot should be compact. Moreover, to avoid assembly and backlash, a monolithic structure is preferred. Positioning resolution should be submicrometric and forces generated should reach several $\mathrm{mN}$ to allow manipulation of various kinds of micro-objects in different environments. The workspace wanted is a square with homogeneous distribution of reachable locations without redundancy in order to simplify the modeling and maximize the reachable space. For mechanical stability and reliability, parallel structures where all bistable modules are fixed to a unique base are privileged. According to all these requirements the design is presented in the following section.

\section{B. Microrobot Design}

This microrobot is made in a monolithic microfabricated structure on SOI (silicon on insulator) wafer, allowing dimensions compatible with confined environments (thickness of $\sim 400 \mu \mathrm{m})$. It could for instance be useful for applications inside a TEM (Transmission Electron Microscope) for which the sample area is very thin. The design chosen for this microrobot is presented in Fig. 2. With respect to the specifications, a flexible structure adapted to microfabrication process is developed to link the displacement of the bistable modules.

The particular kinematics used for the DiMiBot can be parted into elementary structures as the one shown in Fig. 3. This elementary structure is composed of one bistable module connected by a flexible joint (point C) to a first pseudorigid beam $S_{1}$ (oriented in $X_{0}$ direction), which is also connected (at point B) to a second beam $S_{0}$ (oriented in $Y_{0}$ direction). The displacement of the middle point of the $S_{1}$ beam (point D) is generated by the displacement of the bistable module and by the displacement occurring on point $\mathrm{A}$. By associating several of these elementary structures together, the displacement occurring in the point A of one elementary structure is generated by the other parts of the structure. The displacement of each module is then cumulatively transmitted to the end-effector of the microrobot.

\section{DiMiBot's Kinematics}

For digital robots developed at macroscale (VGT, BRAID, ...), the calculation of forward and inverse kinematics is a difficult challenge. This difficulty is due to the generation of a huge number of discrete reachable positions which is difficult to handle for calculation [14]. Several studies were done to overcome this problem, but are dedicated to hyper-redundant manipulators, and make use of workspace densities [11], [12] which is not applicable here. In the case of the DiMiBot which is non-redundant, the modeling of the forward and inverse kinematics is easier.

\section{A. Forward kinematics}

The forward kinematics equation represents the displacement of the end-effector of the robot ( $\left[\begin{array}{ll}\delta_{x} & \delta_{y}\end{array}\right]^{T}$, see Fig. 2) as a function of the states ( 0 or 1 ) of all the bistable modules used. To establish the model, we made several assumptions:

- the beams are rigid (no deformation)

- the rotation occurs at the center of the hinges

- rotations are considered small so that trigonometric functions are linearised ( $\theta$ small and $\alpha$ small, Fig. 4(b))

These assumptions are consistent with the structure because the displacements occuring are very small.

For the calculation of this forward kinematic equation, we will proceed in several steps. The first step consists in representing the displacement of the end effector $E$ as a function 


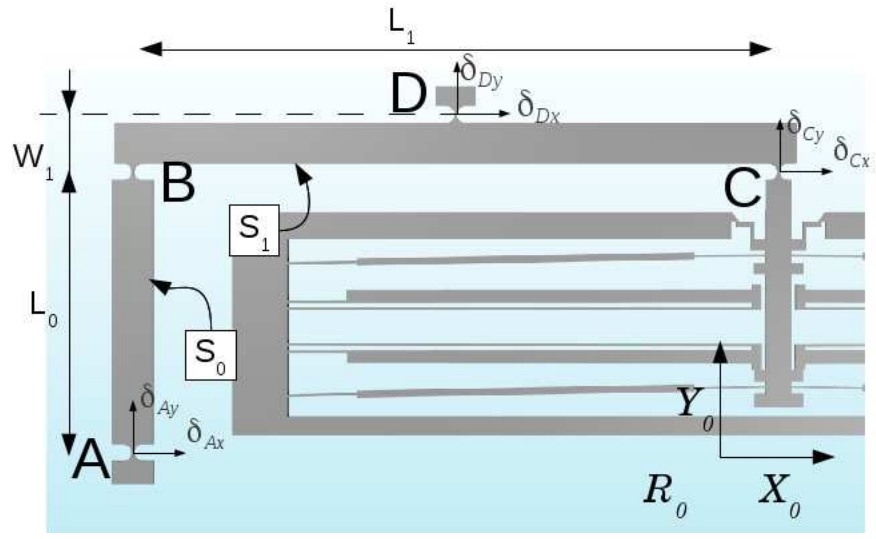

Fig. 3. Elementary structure from the left side of the structure, composed of one bistable module and two beams

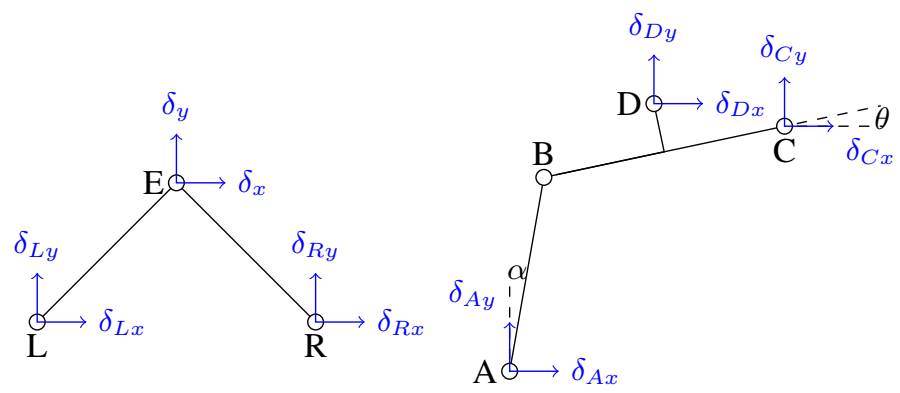

(a) End-Effector

(b) Elementary Structure

Fig. 4. Kinematic schematics of the end-effector part (a), and of the elementary structure (b) constituting the digital microrobot

of the displacements of the two points $L$ and $R$ at the bottom of the two end-effector beams (Fig. 4(a)). The displacement of these two points are noted $\left[\begin{array}{ll}\delta_{L x} & \delta_{L y}\end{array}\right]^{T}$ for the point $L$, and $\left[\begin{array}{ll}\delta_{R x} & \delta_{R y}\end{array}\right]^{T}$ for the point $R$. The relationship between these three points is defined by (1).

$$
\left[\begin{array}{l}
\delta_{x} \\
\delta_{y}
\end{array}\right]=\frac{1}{2}\left[\begin{array}{cccc}
1 & 1 & 1 & -1 \\
1 & 1 & -1 & 1
\end{array}\right] \cdot\left[\begin{array}{l}
\delta_{L x} \\
\delta_{L y} \\
\delta_{R x} \\
\delta_{R y}
\end{array}\right]
$$

The next steps represent the values of the displacements $\left[\begin{array}{ll}\delta_{L x} & \delta_{L y}\end{array}\right]^{T}$ and $\left[\begin{array}{ll}\delta_{R x} & \delta_{R y}\end{array}\right]^{T}$ of points $L$ and $R$ as a function of the states ( 0 or 1 ) of every bistable module. The displacement of point $L$ is expressed as a function of the $b l_{i}$ $\in\{0 ; 1\}\left(0 \leq i \leq N_{1}-1\right.$, numbered from bottom to top, where $N_{1}$ is the number of modules on the left side of the structure). The displacement of point $R$ is expressed as a function of the $b r_{j} \in\{0 ; 1\}\left(0 \leq j \leq N_{2}-1\right.$, numbered from bottom to top, where $N_{2}$ is the number of modules on the right side of the structure). We consider the elementary structure shown in Fig. 3 for the calculation of these displacements. Each elementary structure transmits the displacement of one bistable module to the next elementary structure, except for the ones at the top which make a direct link with the base (see Fig. 2), rigidifying the whole structure. The displacement of all the bistable modules is cumulatively transmitted up to the end-effector. Equation (2) represents the displacement of point $\mathrm{D}\left(\left[\begin{array}{ll}\delta_{D x} & \delta_{D y}\end{array}\right]^{T}\right)$ as a function of the displacement of point $\mathrm{C}\left(\left[\begin{array}{ll}\delta_{C x} & \delta_{C y}\end{array}\right]^{T}\right)$ and of the angle $\theta$, see Fig. 4(b). The displacement of point $D$ is also expressed as a function of the displacement of point $\mathrm{A}\left(\left[\begin{array}{ll}\delta_{A x} & \delta_{A y}\end{array}\right]^{T}\right)$ and of the angles $\theta$ and $\alpha$ in (3).

$$
\begin{gathered}
{\left[\begin{array}{l}
\delta_{D x} \\
\delta_{D y}
\end{array}\right]=\mid \begin{array}{l}
\delta_{C x}-\frac{L_{1}}{2}(\cos (\theta)-1)-W_{1} \sin (\theta) \\
\delta_{C y}-\frac{L_{1}}{2} \sin (\theta)+W_{1}(\cos (\theta)-1)
\end{array}} \\
{\left[\begin{array}{l}
\delta_{D x} \\
\delta_{D y}
\end{array}\right]=\mid \begin{array}{c}
\delta_{A x}+L_{0} \sin (\alpha)+\frac{L_{1}}{2}(\cos (\theta)-1)-W_{1} \sin (\theta) \\
\delta_{A y}+L_{0}(\cos (\alpha)-1)+\frac{L_{1}}{2} \sin (\theta)+W_{1}(\cos (\theta)-1)
\end{array}}
\end{gathered}
$$

Where $L_{1}$ is the length of one beam $S_{1}, W_{1}$ is the width of this beam, and $L_{0}$ is the length of the beam $S_{0}$ (Fig. 3).

With the assumptions mentioned before (small angles approximation), the two equations (2) and (3) can be combined into a single equation (4) representing the displacement of point $D$ as a function of the displacements of points $A$ and $C$. Where the displacement of point $C$ is actually the displacement of the module $\left(\delta_{C x}=0\right.$ and $\left.\delta_{C y}=\Delta \times b l_{i}\right)$.

$$
\left[\begin{array}{l}
\delta_{D x} \\
\delta_{D y}
\end{array}\right]=\left[\begin{array}{cc}
0 & \frac{W_{1}}{L_{1}} \\
0 & \frac{1}{2}
\end{array}\right] \cdot\left[\begin{array}{l}
\delta_{A x} \\
\delta_{A y}
\end{array}\right]+\left[\begin{array}{c}
\frac{-W_{1}}{L_{1}} \\
\frac{1}{2}
\end{array}\right] \cdot \Delta \cdot b l_{i}
$$

To identify the elementary structure of the left side of the microrobot (Fig. 2), we use the same numbering as the corresponding bistable module. We have $A_{i}, B_{i}, C_{i}$ and $D_{i}$ connected to the module $b l_{i}(0 \leq i \leq 2)$, and $A_{3}, B_{3}, C_{3}$ and $D_{3}$ for the top elementary structure (for which $C_{3}$ is fixed, and so is $A_{0}$ ). In that structure $D_{3}=L, A_{3}=D_{2}$, $A_{2}=D_{1}$ and $A_{1}=D_{0}$. Using (4), the displacement of point $L\left(\left[\begin{array}{ll}\delta_{L x} & \delta_{L y}\end{array}\right]^{T}\right)$ is calculated as in (5).

$$
\begin{aligned}
{\left[\begin{array}{l}
\delta_{L x} \\
\delta_{L y}
\end{array}\right] } & =\left[\begin{array}{cc}
0 & \frac{W_{1}}{L_{1}} \\
0 & \frac{1}{2}
\end{array}\right]\left[\begin{array}{l}
\delta_{A 3 x} \\
\delta_{A 3 y}
\end{array}\right] \\
& =\left[\begin{array}{cc}
0 & \frac{W_{1}}{L_{1}} \\
0 & \frac{1}{2}
\end{array}\right]\left(\left[\begin{array}{cc}
0 & \frac{W_{1}}{L_{1}} \\
0 & \frac{1}{2}
\end{array}\right]\left[\begin{array}{l}
\delta_{A 2 x} \\
\delta_{A 2 y}
\end{array}\right]+\left[\begin{array}{c}
\frac{-W_{1}}{L_{1}} \\
\frac{1}{2}
\end{array}\right] \Delta \cdot b l_{2}\right) \\
& =\left[\begin{array}{cc}
0 & \frac{W_{1}}{L_{1}} \\
0 & \frac{1}{2}
\end{array}\right]^{2} \cdot\left[\begin{array}{l}
\delta_{A 2 x} \\
\delta_{A 2 y}
\end{array}\right]+\left[\begin{array}{cc}
0 & \frac{W_{1}}{L_{1}} \\
0 & \frac{1}{2}
\end{array}\right] \cdot\left[\begin{array}{c}
\frac{-W_{1}}{L_{1}} \\
\frac{1}{2}
\end{array}\right] \Delta \cdot b l_{2}
\end{aligned}
$$

The displacement of the point $L$ is then iteratively calculated down to the bottom of the structure, leading to (6) for a robot with $N_{1}$ modules on the left side of the structure $\left(N_{1}=3\right.$ in Fig. 2).

$$
\left[\begin{array}{l}
\delta_{L x} \\
\delta_{L y}
\end{array}\right]=\sum_{i=1}^{N_{1}}\left[\begin{array}{cc}
0 & \frac{W_{1}}{L_{1}} \\
0 & \frac{1}{2}
\end{array}\right]^{i} \cdot\left[\begin{array}{c}
\frac{-W_{1}}{L_{1}} \\
\frac{1}{2}
\end{array}\right] \cdot \Delta \cdot b l_{N_{1}-i}
$$

A similar calculation leads to (7) for the right side of the structure and the displacement of point $R$.

$$
\left[\begin{array}{l}
\delta_{R x} \\
\delta_{R y}
\end{array}\right]=\sum_{j=1}^{N_{2}}\left[\begin{array}{cc}
0 & \frac{-W_{1}}{L_{1}} \\
0 & \frac{1}{2}
\end{array}\right]^{j} \cdot\left[\begin{array}{c}
\frac{W_{1}}{L_{1}} \\
\frac{1}{2}
\end{array}\right] \cdot \Delta \cdot b r_{N_{2}-j}
$$

The displacement of the end-effector can finally be expressed as a function of the state of every bistable module $b l_{i}$ and $b r_{j}\left(0 \leq i \leq N_{1}-1\right.$ and $\left.0 \leq j \leq N_{2}-1\right)$ 


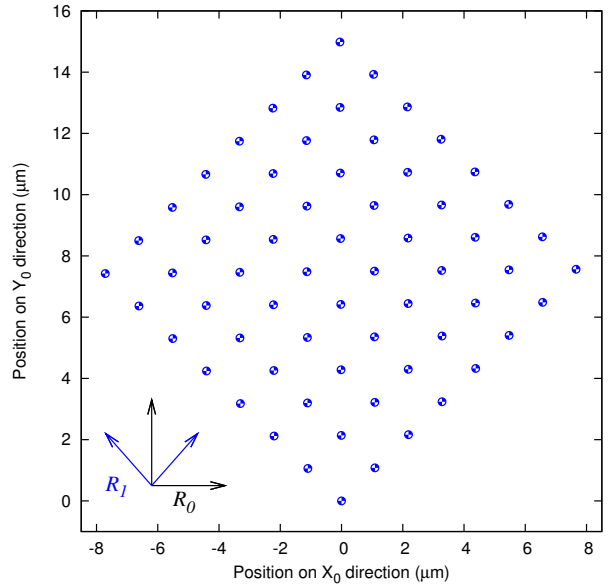

Fig. 5. Workspace generated with a microrobot containing 6 bistable modules for which the displacement between the two states is $\Delta=30 \mu \mathrm{m}$

by combining (1),(6) and (7). The displacement of the endeffector is expressed in (8) when all the bistable modules of the robot generate the same displacement $\Delta$ between their two states.

$\left[\begin{array}{c}\delta_{x} \\ \delta_{y}\end{array}\right]=K \cdot\left[\begin{array}{cccccc}1 & \cdots & \frac{1}{2^{N_{1}-1}} & -1 & \cdots & \frac{-1}{2^{N_{2}-1}} \\ 1 & \cdots & \frac{1}{2^{N_{1}-1}} & 1 & \cdots & \frac{1}{2^{N_{2}-1}}\end{array}\right] \cdot\left[\begin{array}{c}b l_{N_{1}-1} \\ \vdots \\ b l_{0} \\ b r_{N_{2}-1} \\ \vdots \\ b r_{0}\end{array}\right]$

With $K=\frac{\Delta}{4}\left(\frac{1}{2}+\frac{W_{1}}{L_{1}}\right)$ a constant.

The resolution is deduced from (8) (giving the displacement in $\mathcal{R}_{0}$ referential). It is the distance between two neighbor positions in the $X_{1}$ and $Y_{1}$ directions (see Fig. 5). A module $b l_{i}$ generates a displacement on the $X_{1}$ direction $\left(b r_{i}\right.$ in $Y_{1}$ direction). The resolution can be decoupled on each of these directions. The resolution is $r_{x}=\sqrt{2} \frac{\Delta}{4}\left(\frac{1}{2}+\frac{W_{1}}{L_{1}}\right) \frac{1}{2^{N_{1}-1}}$ on the $X_{1}$ direction and is $r_{y}=\sqrt{2} \frac{\Delta}{4}\left(\frac{1}{2}+\frac{W_{1}}{L_{1}}\right) \frac{1}{2^{N_{2}-1}}$ on the $Y_{1}$ direction.

The size of the workspace in the $X_{1}$ direction can be expressed as $2^{N_{1}} \times r_{x}=\sqrt{2} \frac{\Delta}{2}\left(\frac{1}{2}+\frac{W_{1}}{L_{1}}\right)$, which is independent of the number of modules used (independent of $N_{1}$ ). In the $Y_{1}$ direction it is $2^{N_{2}} \times r_{y}$, resulting in the same expression.

\section{B. Workspace Generation}

This particular kinematics generates a two dimensional discrete workspace, meeting the specifications established for the design of the flexible structure. In the case of a robot with 6 bistable modules $\left(N_{1}=N_{2}=3\right)$, like in the kinematic scheme of Fig. 2, the workspace is composed of 64 distinct discrete positions, regularly spread in a Cartesian workspace. All these positions are addressed by a 6 bit binary word composed of the state of each bistable module. By considering a displacement of $\Delta=30 \mu \mathrm{m}$ for each bistable modules (within a structure with dimensions $W_{1}=380 \mu \mathrm{m}, L_{1}=5.8 \mathrm{~mm}$ ), this workspace (Fig. 5) is arranged inside a square of $12 \mu \mathrm{m}$ length with a resolution of $1.5 \mu \mathrm{m}$.

The resolution of the workspace can be improved by increasing the number of bistable modules, leading to submicrometric resolution with high repeatability. For instance by adding two more bistable modules at the bottom of the structure (one on each side of the structure), the number of reachable points becomes $256\left(2^{8}\right)$, and the resolution becomes twice better $(750 \mathrm{~nm})$ while the size of the workspace is unchanged (square of $12 \mu \mathrm{m}$ length). Each time one module is added on one side of the structure, the resolution is divided by two in one direction of the workspace ( $X 1$ direction if it is added on the $X_{0}$ negative half plan side, and $Y 1$ direction if it is on the $X_{0}$ positive half plan side, see Fig. 5).

In order to increase the size of the workspace, two approaches are possible:

- increase the displacement $\Delta$ of every module, while staying in the hypothesis that enable to establish the kinematic model (small angles, ...)

- move the point $L$ along the $-X_{0}$ axis, and point $R$ along the $+X_{0}$ axis, while maintaining an angle of $\frac{\pi}{2}$ between the two end-effector beams (in order to preserve the square shape of the workspace)

\section{Inverse kinematics}

The inverse kinematics model determines the joint parameters that provide a desired position of the end-effector. This modeling is quite complex for digital robots due to the necessary knowledge of all reachable points. The points list can be difficult to handle for calculation when considering robots containing a huge number of binary actuators (possibly over 20). The studies shown in [15], [16], [17] use the high redundancy of digital robots to allow approximations in the calculation of inverse kinematics model.

For the digital microrobot the inverse kinematics model represents the boolean values of all the $b l_{i}$ and $b r_{j}$ for a desired position in the workspace. The inverse kinematics model is established by understanding the pattern of the point distribution inside this workspace. As demonstrated by the forward kinematics model, the modules on the left side of the structure generate a displacement on the $X_{1}$ direction, and the modules on the right side of the structure generate a displacement on the $Y_{1}$ direction. The inverse kinematics model can then be decoupled by considering separately each side of the structure. The points on each direction are furthermore linearly organized on each direction (with a resolution determined by the forward kinematics model), starting from point 0 to point $2^{N_{1}}-1$ or to point $2^{N_{2}}-1$ for $X_{1}$ and $Y_{1}$ directions respectively.

Equation (9) defines the state of every bistable module $i$ $\left(0 \leq i \leq N_{1}-1\right)$ of the left side of the structure and module $j\left(0 \leq j \leq N_{2}-1\right)$ of the right side for any desired position $\left[\begin{array}{ll}x_{d} & y_{d}\end{array}\right]^{\bar{T}}$ (in $\mathcal{R}_{1}$ referential) inside the square workspace.

$$
\left\{\begin{array}{l}
b l_{i}=\neg\left(\left(\operatorname{round}\left(\frac{x_{d}}{r_{x}}\right) \& 2^{i}\right)==0\right) \\
b r_{j}=\neg\left(\left(\operatorname{round}\left(\frac{y_{d}}{r_{y}}\right) \& 2^{j}\right)==0\right)
\end{array}\right.
$$


Where:

- $x_{d}$ is the component along $X_{1}$ axis of the desired position.

- $y_{d}$ is the component along $Y_{1}$ axis of the desired position.

- $b l_{i}$ is the boolean representing the state of the module $i$ on the $X_{0}$ negative half plan of the structure (numbered on the $Y_{0}$ positive direction).

- $b r_{j}$ is the boolean representing the state of the module $j$ on the $X_{0}$ positive half plan of the structure (numbered on the $Y_{0}$ positive direction).

- $r_{x}$ is the resolution of the workspace on the $X 1$ direction.

- $r_{y}$ is the resolution of the workspace on the $Y 1$ direction.

- $\neg$ is the boolean function NOT.

- $\&$ is the bitwise AND function.

\section{DIMENSIONING OF THE FLEXIBLE STRUCTURE}

\section{A. Joint Dimensioning}

This digital microrobot is entirely microfabricated and built in a monolithic structure. The use of flexure hinges is highly used for creating micro/nano-positioning systems with high resolution [18], [19]. Unlike traditional revolute joints, flexure hinges prevent backlash and friction which are the main cause of inaccuracy in positioning and are very troublesome for manipulation tasks at microscale. The flexure hinges, as well as some other parameters of the robotic structure will be characterized in order to provide good behavior of the robot. The results of finite element analysis (FEA) will be used for that purpose.

As the bistable modules were already dimensioned in our previous works [8], [9], the simulation will only deal with the robotic structure. It is composed of a series of two elements which are the pseudo-rigid beams and the flexure hinges (see Fig. 6). The dimensions choice will depend on two simulation results:

- the mechanical stress generated inside the structure by its deformations

- the force applied by the structure onto the bistable modules

While undergoing the displacement of the bistable modules, the robotic structure will deform and tend to return to its rest position (position in which it was after fabrication). A restoring force is then applied by the structure onto each bistable module. In order to produce a stable and robust displacement of the end-effector of the microrobot, and allow its usage in open-loop control (which is the main purpose for designing such digital robots), the force applied on every module should not exceed a given threshold which is the blocking force of the stop blocks $(1.54 \mathrm{mN})$. The stress reached inside the structure should also not exceed a given threshold which is the silicon's rupture stress (assumed to be 1 GPa in this study).

We chose circular flexure hinges in order to mimic the behavior of traditional revolute joints. These circular flexure hinges provide very accurate rotations with an approximately fixed instant center of rotation (consistent with the hypothesis made for the forward kinematics model), which is impossible to get with other hinges such as rectangular hinges or ellipse profile hinges [20]. These hinges will in return produce high

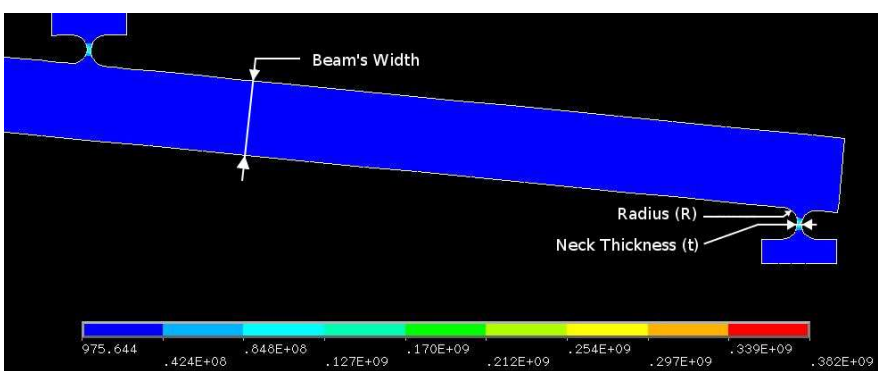

Fig. 6. Elements of the structure studied with the results of the FEA software Ansys
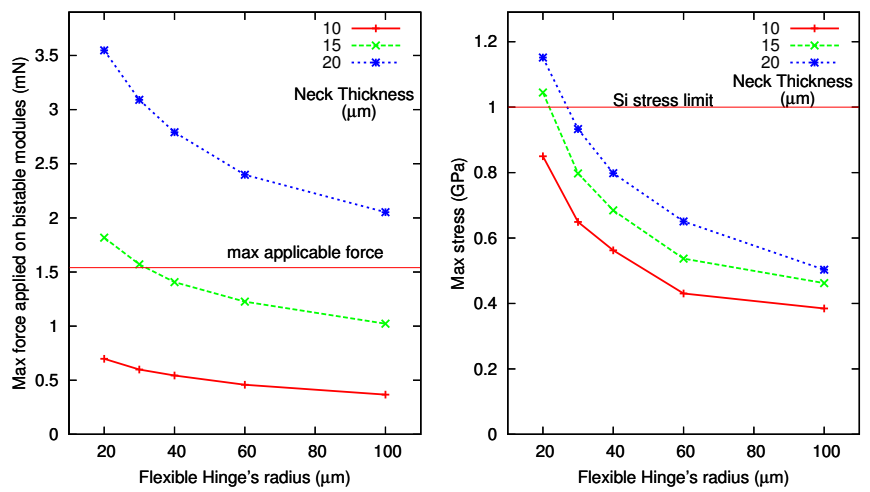

Fig. 7. FEA simulation results: force and stress in the structure depending on the hinge's dimensions (Neck Thickness and Radius)

stress because of the deformation occurring in a very small area.

All the flexure hinges of the flexible structures (represented as circles on the kinematic scheme of Fig. 2) have the same dimensions. In order to find the optimal dimension for these flexure hinges with respect to the constraints mentioned before (stress and force constraints), FEA simulation was made for several dimensions of these flexure hinges.

Fig. 7 represents the value of the maximal force applied on all the bistable modules (left figure), and the maximal stress inside the structure (right figure) for every reachable point of the workspace, and for different dimensions of the hinges (Fig. 6). For hinges' radius from 20 to $100 \mu \mathrm{m}$ (on the abscissa), and neck thickness from 10 to $20 \mu \mathrm{m}$ (the three different curves).

Because of the size uncertainties in the microfabrication processes (about 2 microns due to over-etching), the different elements of the structure should not be too small. If we choose a neck thickness of $15 \mu \mathrm{m}$, and a radius of $60 \mu \mathrm{m}$ for all the flexure hinges of the structure, we ensure the good behavior of this structure.

\section{B. Robustness analysis}

In robotics, robustness analysis deals with the ability of the robot's end-effector to maintain its position when exposed to external perturbations. It is an important property for manipulation tasks, that ensures the good behavior of the manipulator. Robustness it traditionally acquired with control feedback, but in the case of the DiMiBot which is used without 
any sensor we can only consider the mechanical robustness of the robotic structure. When using flexible elements in the structure, external perturbation induces small deformations of the beams, and thus errors in the reached position induces lack of robustness.

The study of the previous section ensured the good behavior of the digital microrobot in standard usage, i.e. with an unladen end-effector, and without any element (objects or obstacles) in the working area. The robustness study of the structure will now consider a perturbation force applied on the endeffector of the structure. In order to be used as a manipulating microrobot used for positioning tasks, it should be robust facing forces of several $\mathrm{mN}$.

The forces applied on every module and the stress inside the structure were simulated for all the positions reachable by the robot while it undergoes an external force from 0 to $10 \mathrm{mN}$. The main consequence of this force is a small displacement of the whole workspace. This study is mainly focused on a robot with beam's width of $300 \mu \mathrm{m}$ and hinges of $15 \mu \mathrm{m}$ thickness and $60 \mu \mathrm{m}$ radius. For this particular design, a force of $5 \mathrm{mN}$ applied on the end-effector induces a workspace displacement of $0.5 \mu \mathrm{m}$. However this displacement can be reduced by increasing the beam's width. For instance this displacement becomes almost ten times smaller with a beam's width of $700 \mu \mathrm{m}$. This small displacement provides compliance to the robot's end-effector, which can be useful for several tasks.

The most constraining characteristic is the force applied on every bistable module. This force should never exceed the limit of $1.54 \mathrm{mN}$. For a structure with beams of $300 \mu \mathrm{m}$ width, the structure can undergo an external force of $3 \mathrm{mN}$ before reaching the blocking force on one of the six bistable modules while with beams of $700 \mu \mathrm{m}$ the external force can not exceed $1.8 \mathrm{mN}$. This means that when applying a strong force (in this case over $3 \mathrm{mN}$ ) on the end-effector of the robot, one of the 6 bistable module could switch back on its own, and the position of the end-effector would be lost. As the first prototype of the digital microrobot will undergo testing of the force it can bear, we chose to use the structure that can handle the strongest external force, i.e. with beam width of $300 \mu \mathrm{m}$.

\section{FEA Simulation vs Kinematic Model}

The simulations made with the FEA software can also be used for a first validation of the geometric model that have been established before. For this, the comparison between the workspace generated by the finite element simulation and the one calculated by the geometric model is made. The results are shown in TABLE I which focuses on the differences between a structure containing beams of $300 \mu \mathrm{m}$ width and a structure with $700 \mu \mathrm{m}$ width beams. The difference between the simulation and the model is established for each of the 64 reachable position. The difference between the two workspaces is $\pm 3.8 \mathrm{~nm}$ in the first case (300 $\mu \mathrm{m}$ width beams), and $\pm 2.2 \mathrm{~nm}$ for the second (700 $\mu \mathrm{m}$ width beams), which is negligible compared to the resolution of the workspace $(\sim 1.5 \mu \mathrm{m})$. This mismatch is due to the deformation of the beams which is not taken into account by the geometric model.

The geometric model established before is then compatible with the simulation results. This modeling is simple (matrix
TABLE I

COMPARISON BETWEEN FEA SIMULATION RESULTS AND MODEL'S CALCULATION

\begin{tabular}{|c||c|c|}
\cline { 2 - 3 } \multicolumn{1}{c|}{} & \multicolumn{2}{c|}{ Beam's width } \\
\cline { 2 - 3 } \multicolumn{1}{c|}{} & $300 \mu \mathrm{m}$ & $700 \mu \mathrm{m}$ \\
\hline \hline Simulations' Resolution & $504 \mathrm{~nm}$ & $567 \mathrm{~nm}$ \\
\hline Model's Resolution & $506 \mathrm{~nm}$ & $567 \mathrm{~nm}$ \\
\hline Difference between the two workspaces & $\pm 3.8 \mathrm{~nm}$ & $\pm 2.2 \mathrm{~nm}$ \\
\hline
\end{tabular}

multiplication), fits well the behavior of the structure and is well adapted for open-loop control.

Finally we decided to microfabricate a DiMiBot composed of beams of $300 \mu \mathrm{m}$ width and circular flexure hinges of $15 \mu m$ neck thickness and $60 \mu m$ radius.

\section{MiCROFABRICATION}

This digital microrobot is a monolithic structure microfabrication on a unique SOI (Silicon On Insulator) wafer. The 4 inch wafer is composed of 3 layers, the bottom one (handle layer) is a silicon layer of $300 \mu \mathrm{m}$ thickness, the middle one (buried oxide 'box' layer) is a silicon oxide $\left(\mathrm{SiO}_{2}\right)$ layer of $2 \mu \mathrm{m}$ thickness, and finally the top one (device layer) is a silicon layer of $100 \mu \mathrm{m}$ thickness. The mobile parts of the digital microrobot are built on the device layer $(100 \mu \mathrm{m}$ thickness) while the fixed base of the robot is composed of the whole wafer (402 $\mu \mathrm{m}$ thickness). The choice to use a SOI wafer was made in order to use the box layer to electrically isolate the aluminium paths used for powering the different bistable modules. It furthermore ensures a constant thickness of the mobile elements of the robot (built on the $100 \mu \mathrm{m}$ device layer) during microfabrication. The microfabrication process is based on the use of chemical etching of silicon and silicon oxide in DRIE (Deep Reactive Ion Etching) machine. The microfabrication process is given in the flow chart shown in Fig. 8. $S 1813, S P R 220-3.0$ and $A Z 9260$ are photoresists used as masks to protect the parts we don't want to remove during the chemical etching process.

During steps (2) to (4) the electrical paths in aluminium are fabricated. In step (2) a $800 \mathrm{~nm}$ layer of aluminium is deposited onto the whole wafer by pulverization, and the photoresist is spincoated onto the aluminium layer. After that (step (3)) the photoresist is patterned and the aluminium etched, before doing the ohmic contact (step (4)) by diffusing the aluminium inside the silicon. Steps (5) to (7) are the etching of the handle layer, while in step (8) we remove the box layer, and finally steps (9) to (11) are the etching of the device layer, and releasing of the microfabricated structure.

A digital microrobot containing 4 bistable modules was microfabricated. The dimensions of this robot are $36.5 \times 24.5 \mathrm{~mm}$ with a thickness of $402 \mu \mathrm{m}$. Each bistable module generates a displacement of $\Delta=30 \mu \mathrm{m}$.

Fig. 9 is a picture of this microrobot, with a zoom on the end-effector during the manipulation of a $150 \mu \mathrm{m}$ diameter glass ball. 


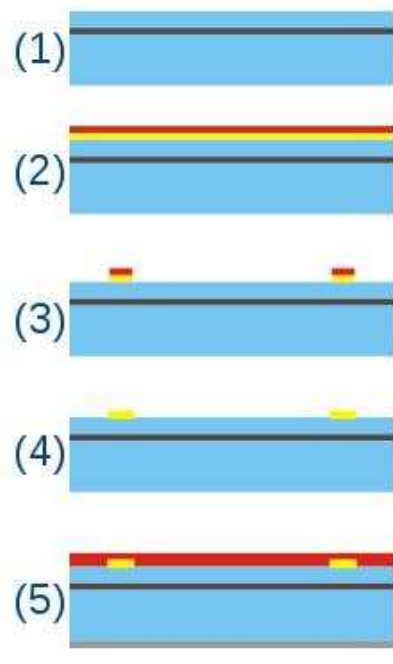

(6)

(7)
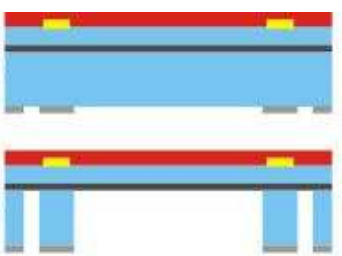

(8)

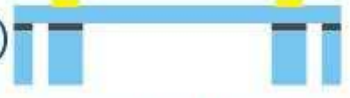

(9)

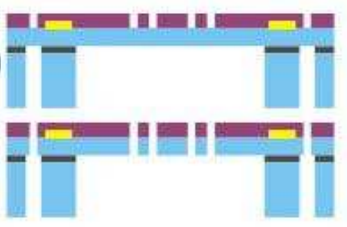

(11)
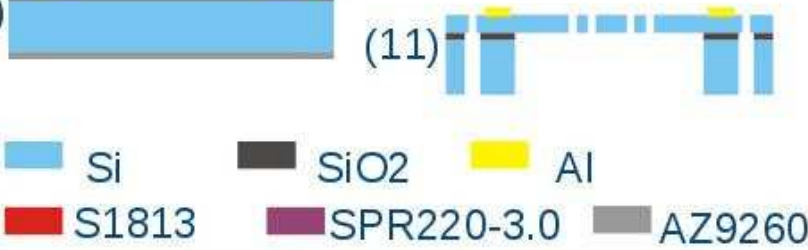

Fig. 8. Flow Chart of the microfabrication process

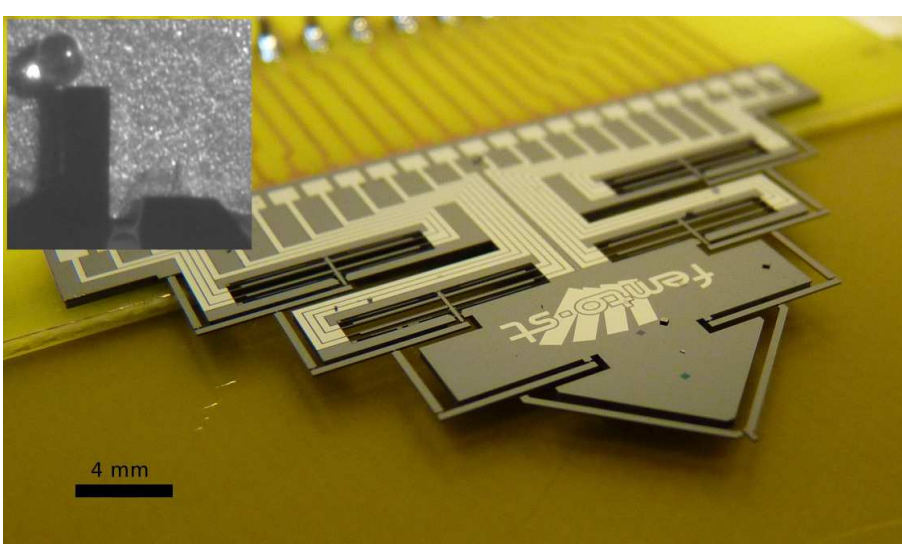

Fig. 9. Picture of the digital microrobot containing 4 bistable modules, and zoom on its end-effector while manipulating a $150 \mu \mathrm{m}$ diameter glass ball

\section{Characterization}

The characterization was done on the digital microrobot containing 4 bistable modules $\left(N_{1}=N_{2}=2\right)$, thus generating a workspace with 16 reachable positions.

\section{A. Necessary Energy for switching modules}

As this microrobot does not need any power to maintain its position, an interesting parameter is the necessary energy for switching the modules. The conditions in which this characterization were made are not optimized, but these results give a first idea concerning the power consumption of this robot.

After microfabrication, the average resistance of one electrothermal actuator is $332.32 \Omega$ (resistivity of the silicon used is $0.02 \Omega \cdot \mathrm{cm}$ ). These actuators are used by pair (in parallel) for switching the bistable module from one state to the other. Each

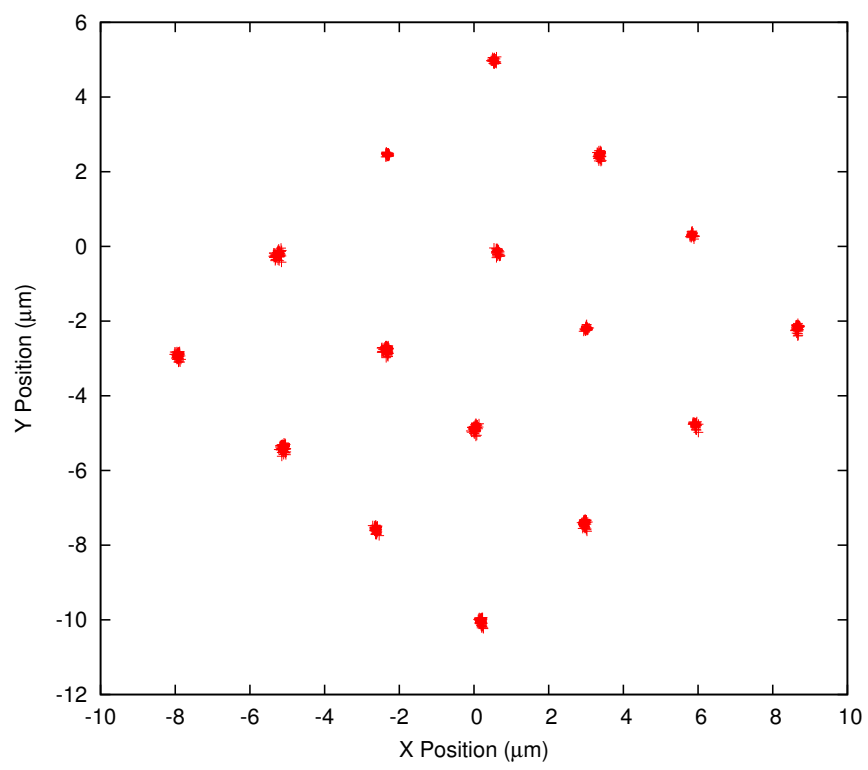

Fig. 10. Scatter plot of all the reached points of the workspace during repeatability characterization

of the two pairs of one bistable module is used for switching in one direction (top or bottom).

The minimum time of input current into the pair of electrothermal actuators needed for switching the module is measured for each state of every bistable module, and the energy is calculated. These results were made for a $16 \mathrm{~V}$ input voltage. The current input inside one pair of electro-thermal actuators is less than $100 \mathrm{~mA}$ for an actuation time lower than $22 \mathrm{~ms}$. The results showed a maximum input energy of $23.5 \mathrm{~mJ}$ for switching a module from one state to the other.

As this energy is only needed when switching module, this makes it a low powered microrobot.

\section{B. Workspace Generation}

This study was made with the Micro System Analyzer $M S A-500$ from Polytec in ambient environment, using the Planar Motion Analysis software. It can measure nanometric size displacements.

Because of uncertainties during the microfabrication process, mainly due to over-etching, the displacement between two states of each bistable module is larger than expected, as we can see in the second column of TABLE II. The third column of this table is the generated displacement at the end-effector of the microrobot for the actuation of each bistable module. This calculation was made with the forward kinematic model by adjusting the value of the displacement of the bistable modules $(\Delta)$. Actually the calculation is slightly different from the kinematic model because the measurement was made on an other point than the center of the endeffector's joint. These results show a good behavior of the complete microrobot. Even if there is a little error between the theoretical workspace and the measured one (error $\sim 5 \%$ ) these results comfort with the established model.

The global generated workspace of this microrobot is represented on Fig. 10. This figure focuses on the repeatability of 
TABLE II

DISPLACEMENT GENERATED AND REPEATABILITY BY EACH OF THE 4 BISTABLE MODULES OF THE DIGITAL MICROROBOT

\begin{tabular}{|c|c|c|c|c|}
\hline \multirow{2}{*}{$\begin{array}{l}\text { Bistable } \\
\text { module }\end{array}$} & \multirow{2}{*}{$\begin{array}{l}\text { Displacement } \\
\text { between } \\
\text { the two states }\end{array}$} & \multicolumn{2}{|c|}{$\begin{array}{l}\text { Displacement at } \\
\text { the end effector }\end{array}$} & \multirow[b]{2}{*}{ Repeatability } \\
\hline & & Theoretical & Measured & \\
\hline$b l_{1}$ & $36.20 \mu \mathrm{m}$ & $7.33 \mu \mathrm{m}$ & $7.71 \mu m$ & $90 \mathrm{~nm}$ \\
\hline$b l_{0}$ & $35.34 \mu \mathrm{m}$ & $3.58 \mu m$ & $3.77 \mu m$ & $100 \mathrm{~nm}$ \\
\hline$b r_{1}$ & $33.6 \mu m$ & $6.8 \mu \mathrm{m}$ & $7.12 \mu \mathrm{m}$ & $75 \mathrm{~nm}$ \\
\hline$b r_{0}$ & $36.50 \mu \mathrm{m}$ & $3.69 \mu m$ & $3.89 \mu \mathrm{m}$ & $60 \mathrm{~nm}$ \\
\hline
\end{tabular}

each position reachable by the microrobot. We can distinguish all the 16 reachable points and their repeatability.

\section{Repeatability study}

The repeatability of a robot represents its ability to return to a given position. Repeatability study have been made for all the bistable modules of this robot. The results are reported in the last column of the TABLE II. This experiment shows a repeatability under $100 \mathrm{~nm}$ while the resolution of this robot is $\sim 3.8 \mu \mathrm{m}$. The results obtained with this first prototype are very encouraging and show the real contribution of designing such a microrobot.

\section{CONCLUSION}

This study opens a new paradigm in the design of microrobots. By making use of bistable modules, it allows the generation of stable and robust discrete reachable positions. The architecture used generates a Cartesian discrete workspace with a resolution of $1.5 \mu \mathrm{m}$ and allow its usage in openloop control. The flexible structure prevents any mechanical drawbacks (backlash, friction, ...) and can withstand external forces up to $3 \mathrm{mN}$. The mechanical stability of the bistable modules furthermore allow energy saving because energy supply is only needed when switching modules, not for maintaining positions. Its small thickness $(\sim 400 \mu \mathrm{m})$ and its open-loop control (no sensor needed) make this robots able to perform micromanipulation tasks in confined environments such as inside a Transmission Electron Microscope. The experiments done on the first prototype (a microrobot with 4 bistable modules) allowed the first validation of a real digital microrobot. The generated workspace of this prototype is consistent with the kinematic models established.

Further studies dealing with actuation energy optimization, and switching dynamic will be performed. The microfabrication process should also be optimized in order to generate displacements closer to the ones expected. Trajectory planning strategies, generally complex for discrete workspaces, will be studied. New architectures generating more complex workspaces (even in three dimensions) will be considered.

\section{ACKNOWLEDGMENT}

The authors would like to thank the Direction Générale de l'Armement (DGA) for its financial support. This work was partly supported by the french RENATECH network and its FEMTO-ST technological facility.

\section{REFERENCES}

[1] M. Rakotondrabe, C. Clévy and P. Lutz, "Hysteresis and vibration compensation in a nonlinear unimorph piezocantilever", in IEEE/RSJIROS (International Conference on Intelligent Robots and Systems), Nice, France, 2008, pp. 558-563

[2] F. Beyeler, A. Neild, S. Oberti, D.J. Bell, Y. Sun, J. Dual and B.J. Nelson, "Monolithically Fabricated Microgripper With Integrated Force Sensor for Manipulating Microobjects and Biological Cells Aligned in an Ultrasonic Field", in Journal of Microelectomechanical Systems, vol. 16, no. 1, 2007, pp. $7-15$

[3] K. Kim, X. Liu, Y. Zhang and Y. Sun, "Nanonewton force-controlled manipulation of biological cells using a monolithic MEMS microgripper with two-axis force feedback", in Journal of Micromechanics Microengineering, vol. 18, no. 5, 2008

[4] http://www.nanotechnik.com/mm3a-em.html

[5] http://www.asyril.ch/products/delta-robots.html

[6] J. Dong, D. Mukhopadhyay and P.M. Ferreira, "Design, fabrication and testing of a silicon-on-insulator (SOI) MEMS parallel kinematics XY stage", in Journal of Micromechanics and Microengineering, vol. 17, no. 6,2007

[7] Y. Tian, B. Shirinzadeh, D. Zhang, X. Liu, and D. Chetwynd, "Design and forward kinematics of the compliant micro-manipulator with lever mechanisms", in Journal of Precision Engineering, vol. 33, no. 4, 2009

[8] Q. Chen, Y. Haddab and P. Lutz, "Digital Microrobotics Based on Bistable Modules : Design of Compliant Bistable Structures", in IEEE/ASME International Conference on Mechatronics and Embedded Systems and Applications, Beijing, ZH, 2008, pp. 36-41

[9] Q. Chen, Y. Haddab and P. Lutz, "Microfabricated Bistable Module for Digital Microrobotics", in Journal of Micro-Nano Mechatronics, Springer Berlin / Heidelberg, vol. 6, 2010, pp. 1-12

[10] G.S. Chirikjian, "A Binary Paradigm for Robotic Manipulators", in IEEE International Conference on Robotics and Automation, San Diego, CA, 1994, pp. 3063-3069

[11] I. Ebert-Uphoff and G.S. Chirikjian, "Efficient Workspace Generation for binary Manipulators with Many Actuators", in Journal of Robotics Systems, vol. 12, 1996, pp. 383-400

[12] D. Lees and G.S. Chirikjian, "An Efficient Method for Computing the Forward Kinematics of Binary Manipulators", in IEEE international conference on Robotics and Automation, Minneapolis, MN, vol. 2, 1996, pp. $1012-1017$

[13] V.A. Sujan, M.D. Lichter and S. Dubowski, "Lightweight Hyperredundant Binary Elements for Planetary Exploration Robots", in IEEE/ASME International Conference on Advanced Intelligent Mechatronics, Como, Italy, vol. 2, 2001, pp. 1273-1278

[14] M.D. Lichter and V.A. Sujan and S. Dubowsky, "Computational Issues in the Planning and Kinematics of Binary Robots", in IEEE International Conference on Robotics and Automation, Washington, DC, USA, 2002, pp. 341-346

[15] I. Ebert-Uphoff and G.S. Chirikjian, 'Inverse Kinematics of Discretely Actuated Hyper-Redundant Manipulators Using Workspace Densities", in IEEE international conference on Robotics and Automation, Minneapolis, MN, vol. 1, 1996, pp. 139-145

[16] D. Lees and G.S. Chirikjian, "A Combinatorial Approach to Trajectory Planning for Binary Manipulators", in IEEE International Conference on Robotics and Automation, Minneapolis, MN, vol. 3, 1996, pp. 2749-2754

[17] J. Suthakorn and G.S. Chirikjian, "A New Inverse Kinematics Algorithm for Binary Manipulators with Many Actuators", in Journal of Advanced Robotics, vol. 15, 2001, pp. 225-244

[18] Y. Koseki, T. Tanikawa, N. Koyachi and T. Arai, "Kinematic Analysis of Translational 3-DOF Micro Parallel Mechanism Using Matrix Method", in IEEE/RSJ International Conference on Intelligent Robots and Systems, Takamatsu, JP, vol. 1, 2000, pp. 786-792

[19] Y.K. Yong, S.S. Aphale and S.O.R. Moheimani, 'Design, identification and control of a flexure-based XY stage for fast nanoscale positioning ", in IEEE journal of Transaction on Technologies, vol. 8, 2009, pp. 46-54

[20] L.L. Howell, Compliant Mechanisms, New York: Wiley, 2001 


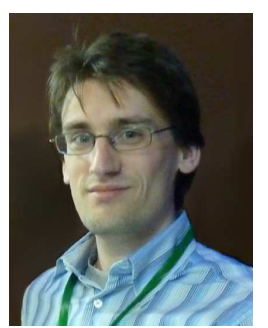

Vincent Chalvet received the Engineering degree in mechatronics in 2008 at ENSMM, Besançon, France, and the M.S. degree in mechatronics and microsystems in 2009 at University of FrancheComté, Besançon, France, where he is currently pursuing the Ph.D. degree. During his studies he focused on robotics and microsystems. He is currently working on the development of novel MEMS digital microrobot for micromanipulation.

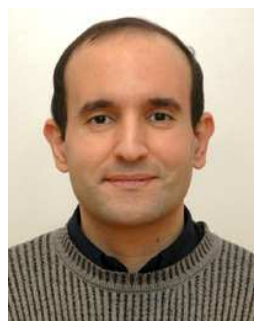

Yassine Haddab received the Engineering degree in electrical engineering at the University of Tizi-Ouzou, Algeria, the M.S. degree at ENSMM, Besançon, France and the Ph.D. degree in 2000 at the University of Franche-Comté, Besançon, France. His work dealt with the design, the modelling and the control of micromanipulation systems. Since 2002 he is an Associate Professor at ENSMM Besançon where he teaches control and microrobotics. His research interests are the design and control of high precision microrobots and microsystems. He also contributes to the development of microfactory concepts and new microrobots architectures.

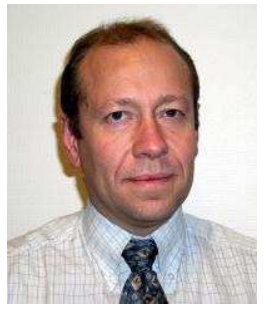

Philippe Lutz joined the University of FrancheComté, Besançon, as Professor in 2002. He was the head of the research group Automated Systems for Micromanipulation and Micro-assembly of the AS2M department of FEMTO-ST from 2005 to 2011. He is currently the Director of the PhD graduate school of Engineering science and $\mathrm{Mi}$ crosystems with more than $400 \mathrm{PhD}$ students. His research activities are focussed on the design and the control of MicroMechatronic Systems. P. Lutz received several awards of IEEE, is author of over 60 refereed publications, serves as associate editor for the IEEE Transaction on Automation Science and Engineering (T-ASE) and is an active member in the IEEE Robotics and Automation Society (RAS) Committee on MicroNano Robotics. He received the Engineer degree from the National School of Mechanics and Microtechnology (ENSMM) in 1990 and the Ph.D. Degree of the University of Franche-Comté in Automation and Computer Science in 1994. He was Associate Professor in the INSA of Strasbourg since 1994 until 2002. 